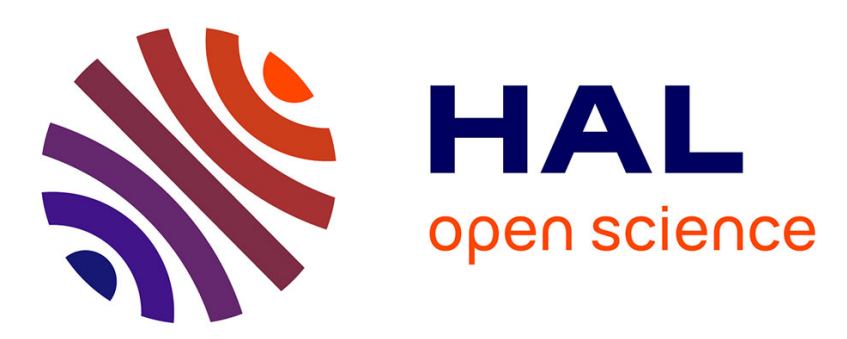

\title{
CLASSIFICATION OF VHR REMOTE SENSING IMAGES USING LOCAL FEATURE-BASED ATTRIBUTE PROFILES
}

Minh-Tan Pham, Sébastien Lefèvre, Erchan Aptoula, Bharath Bhushan Damodaran

\section{To cite this version:}

Minh-Tan Pham, Sébastien Lefèvre, Erchan Aptoula, Bharath Bhushan Damodaran. CLASSIFICATION OF VHR REMOTE SENSING IMAGES USING LOCAL FEATURE-BASED ATTRIBUTE PROFILES. IGARSS 2017, Jul 2017, Fort Worth, United States. hal-01588290

\section{HAL Id: hal-01588290 https://hal.science/hal-01588290}

Submitted on 18 Sep 2017

HAL is a multi-disciplinary open access archive for the deposit and dissemination of scientific research documents, whether they are published or not. The documents may come from teaching and research institutions in France or abroad, or from public or private research centers.
L'archive ouverte pluridisciplinaire $\mathbf{H A L}$, est destinée au dépôt et à la diffusion de documents scientifiques de niveau recherche, publiés ou non, émanant des établissements d'enseignement et de recherche français ou étrangers, des laboratoires publics ou privés. 


\title{
CLASSIFICATION OF VHR REMOTE SENSING IMAGES USING LOCAL FEATURE-BASED ATTRIBUTE PROFILES
}

\author{
Minh-Tan Pham ${ }^{1}$, Sébastien Lefèvre ${ }^{1}$, Erchan Aptoula ${ }^{2}$, Bharath Bhushan Damodaran ${ }^{1}$ \\ ${ }^{1}$ IRISA - Université Bretagne-Sud, UMR 6074, 56000 Vannes, France \\ ${ }^{2}$ Institute of Information Technologies - Gebze Technical University, 41400, Kocaeli, Turkey \\ minh-tan.phameirisa.fr
}

\begin{abstract}
The present paper introduces an extension of attribute profiles (APs) by extracting their local features. The so-called local feature-based attribute profiles (LFAPs) are expected to provide a better characterization of each APs' filtered pixel (i.e. APs' sample) within its neighborhood, hence better deal with local texture information from the image's content. In this work, LFAP is constructed by extracting some simple first-order statistical features of the local patch around each APs' sample such as mean, standard deviation, range, etc. Then, the final feature vector characterizing each image pixel is formed by combining all local features extracted from APs of that pixel. In order to evaluate the effectiveness of the proposed technique, supervised classification using Random Forest classifier is performed on the VHR panchromatic Reykjavik image. Experimental results show that LFAPs can considerably improve the classification accuracy of the standard APs and the recently proposed histogram-based APs.
\end{abstract}

Index Terms - Remote sensing, very high resolution (VHR) images, supervised classification, attribute profiles (APs), local feature-based attribute profiles (LFAPs)

\section{INTRODUCTION}

Morphological attribute profiles (APs) [1] have been widely used to incorporate spectral and spatial information for remote sensing image classification. In the

This work was supported by the French Agence Nationale de la Recherche (ANR) under the reference ANR-13-JS02-0005-01 (Asterix project) and the Région Bretagne grant. It was also supported by the BAGEP Award of the Science Academy and by the Tubitak Grant $115 \mathrm{E} 857$. past few years, a great number of studies have been contributed to exploit and extend the use of APs, especially for classification of optical images. A recent survey of APs and some of their extensions can be found in [2]. In fact, APs provide a multi-level image representation obtained by the sequential application of different filter rules (i.e. attributes) characterizing the size and shape of objects present in the image [1]. By well preserving important spatial properties of regions such as contours, shape, etc. within images, APs become relevant and effective for classification task, in particular for remote sensing images.

Since the direct exploitation of APs for VHR image classification may be insufficient for a complete characterization of textural information, the histogram-based APs (HAPs) have been recently introduced in [3] for an improvement. HAPs are built by concatenating the local histograms of attribute filter responses of each pixel. They have been proved to be more efficient and better deal with local textures in VHR images [3]. However, two limitations of HAPs can be observed involving their very high dimensionality and their high sensitivity to the number of histogram's bins. Therefore in this paper, instead of constructing local histograms, our motivation is to exploit certain statistical features to characterize the local neighborhood around each pixel's attributes. Similar to HAPs, the proposed local feature-based APs (LFAPs) can firstly provide a better description of local textures in VHR images than the standard APs. Then, by using some simple first-order local features, LFAPs can overcome the two aforementioned drawbacks of HAPs. Furthermore, the construction of LFAPs is not limited to the use of first-order statistical features, one can take into consideration other kinds of local features to tackle more complex VHR image scenes. 
The remainder of this paper is organized as follows. The proposed LFAP technique is described in Section 2. In Section 3, supervised classification results of the Reykjavik image yielded by the proposed method and some reference approaches are evaluated and compared in terms of both classification accuracy and time consumption. Section 4 finally concludes the paper and discusses some further work.

\section{METHODOLOGY}

The generation of LFAPs for an image can be divided by two stages. First, the standard APs are built from the image. Then, for each APs' filtered pixel, statistical features of its local neighborhood such as mean, standard deviation, range, etc. are extracted and stacked to form the final LFAP descriptor. We now describe each stage in details.

\subsection{Generation of APs}

Let $X=\left\{x_{i}\right\}_{i=1}^{N}$ be a grayscale image consisting of $N$ pixels. The generation of APs on $X$ is achieved by applying a sequence of attribute thickening $\left\{\phi^{\lambda_{\ell}}\right\}_{\ell=1}^{L}$ and thinning $\left\{\gamma^{\lambda_{\ell}}\right\}_{\ell=1}^{L}$ operations as follows:

$$
\operatorname{AP}(X)=\left\{X^{\phi^{\lambda_{L}}}, \ldots, X^{\phi^{\lambda_{1}}}, X, X^{\gamma^{\lambda_{1}}}, \ldots, X^{\gamma^{\lambda_{L}}}\right\}
$$

where $X^{\phi^{\lambda} \ell}=\left\{x_{1}^{\phi^{\lambda} \ell}, x_{2}^{\phi^{\lambda_{\ell}}}, \ldots, x_{N}^{\phi^{\lambda_{\ell}}}\right\}$ is the filtered image obtained by applying the attribute thickening $\phi^{\lambda}$ with regard to the threshold $\lambda_{\ell}$. Similar explanation is made for $X^{\gamma^{\lambda} \ell}$. For more details about this AP computation, readers are referred to papers $[1,2]$.

It should be noted that for each pixel $x_{i} \in X, i=$ $1, \ldots, N$, the following feature vector can be considered as its AP descriptor which has been commonly used for classification task:

$$
\operatorname{AP}\left(x_{i}\right)=\left\{x_{i}^{\phi^{\lambda_{L}}}, \ldots, x_{i}^{\phi^{\lambda_{1}}}, x_{i}, x_{i}^{\gamma^{\lambda_{1}}}, \ldots, x_{i}^{\gamma^{\lambda_{L}}}\right\} .
$$

\subsection{Construction of LFAPs}

Due to the increase in spatial resolution, the appearance of geometrical and textural information in VHR remote sensing images becomes more and more significant. Hence, classification task should take more into account textural features when dealing with VHR image data.
It should be noted that textures are generally not derived from a single image pixel, but from a local neighborhood around it. That is the reason why the direct application of AP feature vector in Eq. (2) for classification may be not sufficient to account for textures in VHR images. To this end, replacing the standard AP's filtered pixel by the statistical features extracted from its local neighborhood appear to be a good strategy to resolve this issue.

Local feature extraction has been one of the main approaches for texture analysis for decades [4]. Firstorder, second-order or higher order statistical features extracted from the local patch can describe the roughness, regularity, homogeneity, contrast, etc. of the related texture [4]. An important remark is that AP images mostly consist of homogeneous (flat) regions. Hence, only first-order local features such as mean, range, variance or standard deviation can be sufficient to model texture information. That is why we recommend to exploit only first-order features to construct LFAPs. Particularly in this work, we propose to use the mean and the range thanks to their fast computation and good representation of smooth textures.

We define the LFAP feature vector for each pixel $x_{i} \in X$ as follows:

$$
\operatorname{LFAP}\left(x_{i}\right)=\left\{\operatorname{LFAP}_{\mu}\left(x_{i}\right), \operatorname{LFAP}_{r}\left(x_{i}\right)\right\}
$$

in which

$$
\begin{array}{r}
\operatorname{LFAP}_{\mu}\left(x_{i}\right)=\left\{\mu\left(x_{i}^{\phi^{\lambda}}\right), \ldots, \mu\left(x_{i}^{\phi^{\lambda_{1}}}\right), \mu\left(x_{i}\right),\right. \\
\left.\mu\left(x_{i}^{\gamma^{\lambda_{1}}}\right), \ldots, \mu\left(x_{i}^{\gamma^{\lambda_{L}}}\right)\right\} \\
\operatorname{LFAP}_{r}\left(x_{i}\right)=\left\{r\left(x_{i}^{\phi^{\lambda_{L}}}\right), \ldots, r\left(x_{i}^{\phi^{\lambda_{1}}}\right), r\left(x_{i}\right),\right. \\
\left.r\left(x_{i}^{\gamma^{\lambda_{1}}}\right), \ldots, r\left(x_{i}^{\gamma^{\lambda_{L}}}\right)\right\}
\end{array}
$$

where $\mu\left(x_{i}\right)$ and $r\left(x_{i}\right)$ are the mean and range values extracted from the local patch $P\left(x_{i}\right)$ of size $w \times w$ from the image $X$ :

$$
\begin{gathered}
\mu\left(x_{i}\right)=\frac{1}{w^{2}} \sum_{x_{j} \in P\left(x_{i}\right)} x_{j}, \\
r\left(x_{i}\right)=\max \left(P\left(x_{i}\right)\right)-\min \left(P\left(x_{i}\right)\right) .
\end{gathered}
$$

We note that similar calculations are done to extract the mean and range values from every other APs' filtered image (i.e. every $\left.X^{\phi^{\lambda_{\ell}}}, X^{\gamma^{\lambda_{\ell}}}, \ell=1, \ldots, L\right)$.

It should be remarked that the histogram-based attribute profiles (HAPs) proposed in [3] can be considered as a specific case of LFAPs when local histogram 
features are extracted to construct the descriptor:

$$
\begin{aligned}
\operatorname{HAP}\left(x_{i}\right) & =\operatorname{LFAP}_{h}\left(x_{i}\right) \\
& =\left\{h\left(x_{i}^{\phi^{\lambda_{L}}}\right), \ldots, h\left(x_{i}^{\phi^{\lambda_{1}}}\right), h\left(x_{i}\right),\right. \\
& \left.h\left(x_{i}^{\gamma^{\lambda_{1}}}\right), \ldots, h\left(x_{i}^{\gamma^{\lambda_{L}}}\right)\right\}
\end{aligned}
$$

where $h\left(x_{i}\right)$ is the local histogram constructed from the local patch $P\left(x_{i}\right)$ from $X$.

The extraction of local histogram requires another parameter: the number of bins (namely $n b$ ). Hence, unlike the case of mean and range features which are scalar values, each $h\left(x_{i}\right)$ is a vector of $n b$ features, which makes the HAPs become very high dimensional. Moreover, their high sensitivity to $n b$ is another drawback of this approach. We observe how the proposed LFAPs outperform the standard APs as well as the HAPs during our experimental study in the next section.

\section{EXPERIMENTAL STUDY}

\subsection{Data description and experimental setup}

Our experiments were conducted on the VHR panchromatic image of Reykjavik, Iceland, acquired by the IKONOS Earth imaging satellite with a spatial resolution of 1 meter. The image size is $976 \times 640$ pixels. A thematic ground-truth of six classes is available for classification evaluation. The image and its ground-truth are shown in Figs. 1(a) and (b). It is worth noting that a pansharpened multispectral (MS) image is also available for this scene. However, in this paper, we would like to demonstrate the effectiveness of the proposed approach by only exploiting the panchromatic image. MS bands can be also used as complementary information to improve classification performance in further work.

Supervised classification results of the proposed LFAPs will be evaluated and compared to those yielded by the standard APs and the HAPs. To construct the APs, three common attributes were considered including the area, the standard deviation and the moment of inertia. The threshold values were set to be similar to previous studies on the same data [5]. For classification stage, the Random Forest was employed by setting the number of trees equal to 200 . We randomly selected $1 \%$ of each class for training and the rest for testing. Then, another experiment scenario was conducted using $10 \%$ of training samples. All experiments were run 10 times to report the mean and standard deviation of classifica- tion results, in terms of overall accuracy (OA), average accuracy (AA) and Kappa coefficient.

\subsection{Results}

Fig. 1 and Tab. 1 show the classification performance of the proposed LFAPs compared to the pixel intensitybased approach (PAN), APs and HAPs. For LFAPs and HAPs, the local patch size was set to $7 \times 7$. HAPs were also tested with different number of bins.

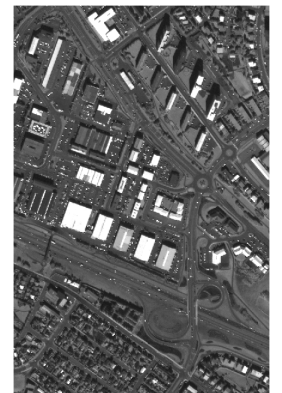

(a) Panchromatic image

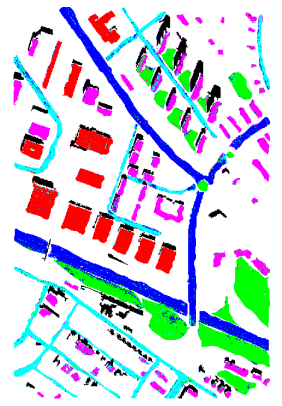

(d) AP

$(\mathrm{OA}=91.68 \%)$

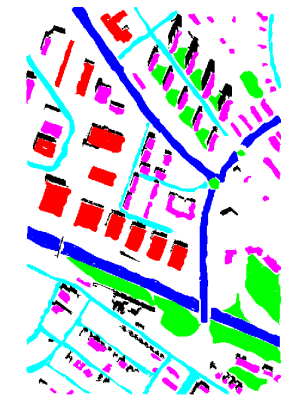

(b) Ground truth

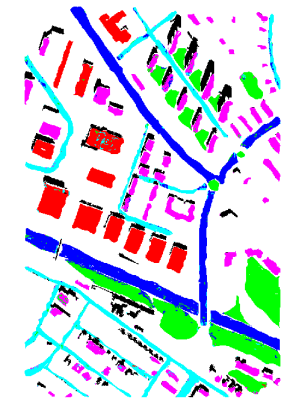

(e) HAP $(n b=9)$

$(\mathrm{OA}=95.83 \%)$

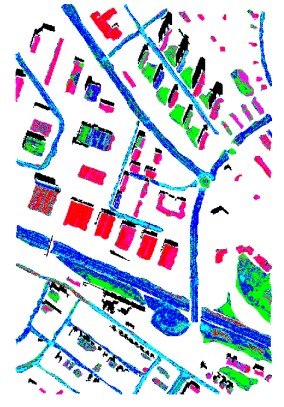

(c) PAN

$(\mathrm{OA}=52.62 \%)$

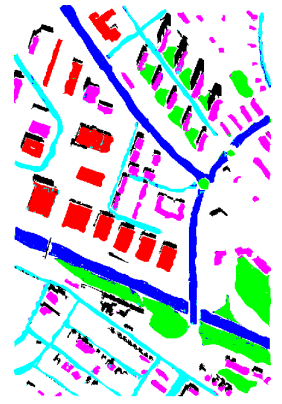

(f) LFAP

$(\mathrm{OA}=97.18 \%)$
Fig. 1: Classification results of the Reykjavik image obtained by different methods ( $10 \%$ training samples)

From the figure, classification result yielded by APs (Fig. 1(d)) still contains some noise which is mostly reduced from the results of HAPs and LFAPs (Figs. 1(e) and 1(f)). The use of local patch's features instead of single APs' sample helps to provide smoother results. For quantitative evaluation, Tab. 1 shows that the highest OA has been achieved by the proposed LFAP method (i.e. $91.03 \%$ and $97.18 \%$ in case of using $1 \%$ and $10 \%$ of training samples, respectively). Our technique has improved more than $6 \%$ of OA from the standard APs. Then, compared to HAPs, a superiority of $2.78 \%$ and $1.05 \%$ in OA is provided by LFAPs. We also remark that the performance of HAPs is quite sensitive to $n b$ (i.e. 
OA is low with $n b=5$, considerably increased when $n b=7$ but then decreased when $n b=9$ ). Readers can also observe this behavior from the experimental study of paper [3] .

\begin{tabular}{|l||c|c|c|}
\hline Method & OA $(\%)$ & AA $(\%)$ & kappa $\times \mathbf{1 0 0}$ \\
\hline \multicolumn{4}{|c|}{ 1\% of samples used for training } \\
\hline PAN & $50.93 \pm 0.44$ & $52.61 \pm 0.48$ & $40.67 \pm 0.54$ \\
\hline AP & $84.81 \pm 0.51$ & $85.23 \pm 0.48$ & $81.65 \pm 0.62$ \\
\hline HAP(nb=5) & $84.91 \pm 0.42$ & $85.16 \pm 0.43$ & $81.77 \pm 0.51$ \\
\hline HAP(nb=7) & $88.25 \pm 0.30$ & $88.59 \pm 0.32$ & $85.81 \pm 0.36$ \\
\hline HAP(nb=9) & $87.40 \pm 0.31$ & $87.71 \pm 0.31$ & $84.79 \pm 0.38$ \\
\hline LFAP & $\mathbf{9 1 . 0 3} \pm \mathbf{0 . 4 4}$ & $\mathbf{9 1 . 1 2} \pm \mathbf{0 . 4 4}$ & $\mathbf{8 9 . 3 8} \pm \mathbf{0 . 5 3}$ \\
\hline \multicolumn{5}{|c||}{ 10\% of samples used for training } \\
\hline PAN & $52.62 \pm 0.13$ & $54.34 \pm 0.12$ & $42.74 \pm 0.14$ \\
\hline AP & $91.68 \pm 0.12$ & $90.84 \pm 0.11$ & $89.96 \pm 0.15$ \\
\hline HAP(nb=5) & $92.93 \pm 0.07$ & $93.06 \pm 0.07$ & $91.46 \pm 0.09$ \\
\hline HAP(nb=7) & $96.13 \pm 0.07$ & $96.28 \pm 0.07$ & $95.33 \pm 0.08$ \\
\hline HAP(nb=9) & $95.83 \pm 0.10$ & $95.92 \pm 0.10$ & $94.96 \pm 0.12$ \\
\hline LFAP & $\mathbf{9 7 . 1 8} \pm \mathbf{0 . 1 3}$ & $\mathbf{9 7 . 1 9} \pm \mathbf{0 . 1 3}$ & $\mathbf{9 6 . 6 0} \pm \mathbf{0 . 1 6}$ \\
\hline
\end{tabular}

Table 1: Classification accuracy of Reykjavik data obtained by different methods

We now observe in Tab. 2 the dimensionality of AP, LFAP and HAP descriptors and the calculation time for their extraction as well as for classification stage (implementation on Matlab using a PC $3.4 \mathrm{GHz}, 16 \mathrm{~GB}$ RAM). Since the mean and range features are used to form LFAP descriptor, its extraction time is quite low. Then, its dimension is twice of that of APs. Meanwhile, the length of HAP vector involves a multiplication factor of $n b$ and becomes very huge when a high value of $n b$ is set. This issue leads to a major limitation of HAPs in terms of high storage requirement as well as high computational cost for classification phase.

\begin{tabular}{|l|cr|rr|}
\hline \multirow{2}{*}{ Method } & \multicolumn{2}{|c|}{ Feature extraction } & \multicolumn{2}{c|}{ Classification } \\
\cline { 2 - 5 } & Dimension & Time & Training & Testing \\
\hline \hline AP & 63 & $46.9 \mathrm{~s}$ & $38.7 \mathrm{~s}$ & $3.2 \mathrm{~s}$ \\
\hline LFAP & 126 & $1 \mathrm{~m} 35.6 \mathrm{~s}$ & $1 \mathrm{~m} 25.3 \mathrm{~s}$ & $3.9 \mathrm{~s}$ \\
\hline HAP $(\mathrm{nb}=5)$ & 315 & $3 \mathrm{~m} 57.5 \mathrm{~s}$ & $3 \mathrm{~m} 38.1 \mathrm{~s}$ & $6.3 \mathrm{~s}$ \\
\hline HAP $(\mathrm{nb}=7)$ & 441 & $4 \mathrm{~m} 01.2 \mathrm{~s}$ & $4 \mathrm{~m} 52.3 \mathrm{~s}$ & $7.0 \mathrm{~s}$ \\
\hline HAP $(\mathrm{nb}=9)$ & 567 & $4 \mathrm{~m} 07.1 \mathrm{~s}$ & $6 \mathrm{~m} 20.4 \mathrm{~s}$ & $7.8 \mathrm{~s}$ \\
\hline
\end{tabular}

Table 2: Comparison of feature dimension and calculation time of AP, LFAP and HAP methods ( $10 \%$ training samples)

\section{CONCLUSION}

The local feature-based attribute profiles (LFAPs) have been proposed to improve the classification performance of VHR remote sensing images. For better dealing with spatial and texture information, statistical features of local patch are extracted to replace each APs' filtered pixel. In this work, two simple first-order features including the mean and range have been exploited and proved to be relevant for characterizing smooth textures from AP filtered images. Our experimental study has shown superior performance of LFAPs compared the standard APs and the histogram-based approach.

The construction of LFAPs is not limited to the use of mean and range features. Any other local features can be extracted to tackle more complex VHR image scenes in future work. Also, the concept of LFAPs can be applied to the extended APs [6] for hyperspectral image classification as well as to the recently proposed selfdual APs [5] which has been proved to outperform APs.

\section{ACKNOWLEDGEMENT}

The authors would like to thank Prof. Jon Atli Benediktsson for providing the Reykjavik image and Dr. Mauro Dalla Mura for providing the Matlab code of APs.

\section{REFERENCES}

[1] M. Dalla Mura, J. A. Benediktsson, N. Waske, and L. Bruzzone, "Morphological attribute profiles for the analysis of very high resolution images," IEEE Trans. Geosci. Remote Sens., vol. 48, no. 10, pp. 3747-3762, 2010.

[2] P. Ghamisi, M. Dalla Mura, and J. A. Benediktsson, "A survey on spectral-spatial classification techniques based on attribute profiles," IEEE Trans. Geosci. Remote Sens., vol. 53, no. 5, pp. 2335-2353, 2015.

[3] B. Demir and L. Bruzzone, "Histogram-based attribute profiles for classification of very high resolution remote sensing images," IEEE Trans. Geosci. Remote Sens., vol. 54, no. 4, pp. 2096-2107, 2016.

[4] A. Materka et al., "Texture analysis methods-A review," Technical university of lodz, institute of electronics, COST B11 report, Brussels, pp. 9-11, 1998.

[5] G. Cavallaro, M. Dalla Mura, J. A.Benediktsson, and A. Plaza, "Remote sensing image classification using attribute filters defined over the tree of shapes," IEEE Trans. Geosci. Remote Sens., vol. 54, no. 7, pp. 3899-3911, 2016.

[6] M. Dalla Mura, J. A. Benediktsson, B. Waske, and L. Bruzzone, "Extended profiles with morphological attribute filters for the analysis of hyperspectral data," Int. J. Remote Sens., vol. 31, no. 22, pp. 5975-5991, 2010. 